\title{
Food Selectivity, Mealtime behavior, Weight status and Dietary intake in Children and adolescent with Autism
}

\author{
Kshitiz Upadhyay Dhungel ${ }^{1}$, Sukriti Ghimire² \\ Department of Nutrition and Dietetics \\ College of Applied Food and Dairy Technology
}

(CAFODAT)

1Professor \& Supervisor, Department of Physiology, Janaki Medical College, Janakpurdham, Nepal 2MSc, student, Nutrition and Dietetics, CAFODAT

\begin{abstract}
Background and Objectives: Food selectivity is a common parental concern in childhood that can become problematic and lead to issues such as nutritional deficits that may impair growth and development. Considering the problem of food selectivity and meal time behaviors in the children with autism, this study was undertaken to assess their food selectivity, mealtime behavior problems, nutrient intake and weight status of children and adolescent with autism of Autism Care Nepal Society.

Material and methods: This is a descriptive cross sectional study carried out in Autism children and adolescent aged 3-19 years in Autism Care Nepal Society. The sampling method was purposive sampling by total enumeration method. A pretested structured questionnaire was used to collect data using a face-to-face interview. Data were entered and analyzed in Microsoft Excel. Descriptive analysis was done to determine the socio-demographic characteristics. Chi-square test was done to determine the association of different independent variables with the BMI.

Results: Out of 70 participants, 49 were male and 29 females. Among 70 participants, about $4 \%$ individuals were underweight, $39 \%$ normal and $57 \%$ were overweight or obese ( $\geq 85$ th percentile). Out of this $36 \%$ were obese ( $\geq 95$ th percentile). Out of 70 participants, 36 individuals $(51.43 \%)$ were food selective while 34 individuals (48.57\%) were not food selective that $51.43 \%$ of autism children and teenagers do not have problematic meal time behavior while $48.57 \%$ of them have problematic meal time behavior.

Conclusion: There was no significant association between age, gender, family income, physical activity, food selectivity and mealtime behavior with BMI ( $p>0.05)$ among autistic children. However, food selectivity of the autistic participants showed a significant association with obesity. There was significant difference in mean calorie intake by all the age groups than the calorie set intake by Indian council of Medical Research (ICMR) as p-value was $<0.05$ in all age group.
\end{abstract}

Key words: Autism, ASD, CWA, Overweight, Obese, BMI 


\section{INTRODUCTION}

Autism is a neuro-developmental syndrome that is defined by deficits in communication and by unusual restricted and repetitive behaviors. This disorder usually begins in infancy, or in first three years of life at the latest [1]. Autism Spectrum Disorders (ASD) have been reported to affect one in 68 children in the United States and one in 42 boys ; boys are five times more likely to be diagnosed with an ASD when compared to girls [2]. It is estimated that autism has increased threefold since the 1970s and that current rates are around 5-10 cases per 10,000 in the general population.

Children with autism spectrum disorder (ASD) are more likely to experience feeding problems compared to typically developing (TD) children, and food selectivity is often reported as a common cause of their feeding problems [3, 4]. The prevalence of problem eating behaviors in children with autism has been estimated to range between $46 \%$ and $89 \%$.It is postulated that these challenging or problem eating behaviors, which can severely compromise nutrient intake [5]. Children with autism are reported as being more likely to exhibit food selectivity behaviors (ie, by texture, food group,type, etc), food neophobia (fear of trying unfamiliar foods), and obsessive eating habits than children with other developmental disabilities or typically developing children $[3,5]$.

Children with autism may have sensory hypersensitivities and reject food due to an aversion to texture, temperature or other characteristics of the foods. They also have limited food preferences. Problematic eating behaviors include smelling and throwing food, mouthing objects and unwillingness to try new food. Feeding problems may be mild to severe, where child may prefer only one or two foods, or only one type of texture. This restriction becomes a threat to overweight, obesity as it limits a variety of fruits, vegetables, lean meats that children with an ASD consume. The child may also feel uncomfortable or threatened or over stimulated due to anxiety, and may often act out, throwing food, crying, spitting or fussing in general. Clinical trials have documented the frequency of problem-eating behaviors as high as $72 \%-77 \%$ of children with ASD [6]. Although picky eating is not uncommon in young children, it appears that children with an ASD may be more selective and it may extend past childhood. There are numerous case reports and considerable anecdotal evidence that supports that food selectivity in children with an ASD is a problem [7]. These behaviors compromise nutritional status and optimal growth in children with autism. Emerging evidence suggests the feeding problems and dietary patterns associated with autism may place this population at risk for long-term medical complications, including poor bone growth, obesity and other diet-related diseases (e.g., cardiovascular disease) in adolescence or adulthood.

Although it is not unusual for typically developed children to present signs of food selectivity, selective eating has a much higher prevalence in children on the autism spectrum and it is usually presented differently in this population [7]. Children with ASD are five times more likely to have mealtime challenges such as tantrums and extreme food selectivity [4]. Various studies found that food selectivity and meal time behavior problems in children show the prevalence of $40-70 \%$ in Unites States of America [4, 7-9]. Mealtime behavior problems was shown to be $30-50 \%$ in the 
studies conducted in Asian countries like Hongkong-China, India $[10,11]$.

Food refusal and selectivity, coupled with potential gastrointestinal concerns, are likely to place an individual at risk of nutrient insufficiency and thus nutrition related diseases. Therefore, the assessment of the mealtime behavior problems, food selectivity pattern and their relation with the weight status of children with autism needed to be done as they have multiple risk factors that could amplify nutritional deficiencies.

Accurate understanding of nutritional risk associated with food selectivity with autism is important to dietitians who are responsible for dietary surveillance as well as to the parents and caregivers of children with autism. Considering the problem of food selectivity and meal time behaviors in the children with autism, this study was undertaken to assess their food selectivity, mealtime behavior problems, nutrient intake and weight status of children with autism of Autism Care Nepal Society.

\section{MATERIAL AND METHODS}

This is a descriptive cross sectional study carried out in Autism children aged 3-19 years in contact with Autism Care Nepal Society (ACNS) whose family agrees to participate were included in the study.Data was collected using semi- structured questionnaire, which was prepared after proper literature review. It consists of information about socio-demographic and socio-economic variables, list of foods for food selectivity, Brief Autism Mealtime Behavior Inventory (BAMBI) [12] questions for assessing meal time problems and questions that are relatable to autism. The questionnaire was tested by pilot study and was modified.
Operational definition: Food refusal is defined as the percentage of foods the child refused to eat among those foods that the child was offered [8]. The absolute number of foods that a child refused will also be examined and also the absolute number of food items not offered. The children are classified as having "high food selectivity" if they refused $>33 \%$ of foods offered to them.

Food selectivity: The three domains (refusal, high frequency single food intake and limited repertoire) can be used together or separately to characterize an individual's food selectivity. For the present analysis, the food refusal domains elected as the measure of food selectivity.

Mealtime behavior: A number of different tools have been used to measure difficult mealtime behaviors in children with ASD. Questionnaire for assessing meal time behavior will be based on (BAMBI) [12].

The Brief Autism Mealtime Behavior Inventory (BAMBI) is the standardized measure developed for mealtime behavior specific to the ASD population. It consists of 18 items, and is a parent self-report questionnaire. A frequency score can be calculated using the total score of the 18 Likert responses. Higher scores represent more problematic mealtime behaviour. The BAMBI has three domains: 'Limited Variety', 'Food Refusal' and 'Features of Autism'. The 'Limited Variety' domain consists of eight items related to limited food preferences, 'Food Refusal' has five items related to rejection of food, and the 'Features of Autism' has five items related to behavioral characteristics or associated features of Autism. It indicates how often their child engages in a particular eating behavior with response options ranging from 1 for 'never' to 
5 for 'always' with a neutral midpoint. A cut off score was 34 [12]. Nutrient intake was observed from 24 hours dietary recall method. The information was further used to calculate total kilocalories and macronutrient intakes.

Study variables: Body Mass Index (BMI) was dependent variable where as Socio-economic variable(family income), Demographic variables (age and gender), Physical Activity, Food selectivity and Meal time behavior was independent variables.

Data were entered and analyzed in MS Excel. Anthropometric data (weight and height) were used for further computing BMI percentile. Twenty four hour dietary recall, daily intake of food variety of food was calculated manually. It was followed through considering the household measurement through simple tea glass of $130 \mathrm{ml}$ the principles and guidelines made by the Tribhuvan University Teaching Hospital (TUTH) calculated manually. The qualitative data were presented on descriptive statistics using bar diagram, pie chart, percentage and table. The relationship between dependent and independent variables were shown by using chi-square test. The association between dependent and independent variables was shown by chi-square test. Standard and well controlled instruments was used for the measurement of the weight and height to reduce error of measurement. Questionnaire was pre-tested for maintaining accuracy.

Ethical consideration: The study has been conducted only after getting approval letter from the Research Committee of College of Applied Food and Dairy Technology (CAFODAT). Informed consent: The participant for this study had been clarified about the aim of the study and its importance. Without the consent of the participants, they had not been included in the study. The respondents' personal information had been kept confidential.

\section{RESULTS}

The mean age of the participants was $8.95 \pm$ 2.34 years. Table 1 shows the age distribution of the participants of the study. As per the total sample collected and studied, it was found that majority of the participants are first child in the family which stands at $68 \%$. Following that, around 29\% family reported that patients with autism are second child in their family. Third child with autism were $2.86 \%$.

Table 1: Age distribution among the children with autism

\begin{tabular}{lll}
\hline Age & Frequency & Percent\% \\
\hline $\mathbf{1 - 3}$ & 2 & 2.86 \\
$\mathbf{4 - 6}$ & 25 & 35.72 \\
$\mathbf{7 - 9}$ & 16 & 22.86 \\
$\mathbf{1 0 - 1 2}$ & 12 & 17.14 \\
$\mathbf{1 3 - 1 5}$ & 8 & 11.42 \\
$\mathbf{1 6 - 1 9}$ & 7 & 10 \\
\hline Total & 70 & 100 \\
\hline
\end{tabular}

\section{Characteristics related to Autism}

Physical activity: The current study showed that find majority of the children exhibit moderate level of physical activity. However, hyperactive kids are also in big percentage which is $32 \%$. Although it is generally assumed that most of the autism kids and teenagers are hyperactive by nature due to ASDS, but here our finding shows that out of the total sample size $47 \%$ of them are 
moderate in physical activities and $32 \%$ are hyper active. Slowed down kids are only $20 \%$ in this case.

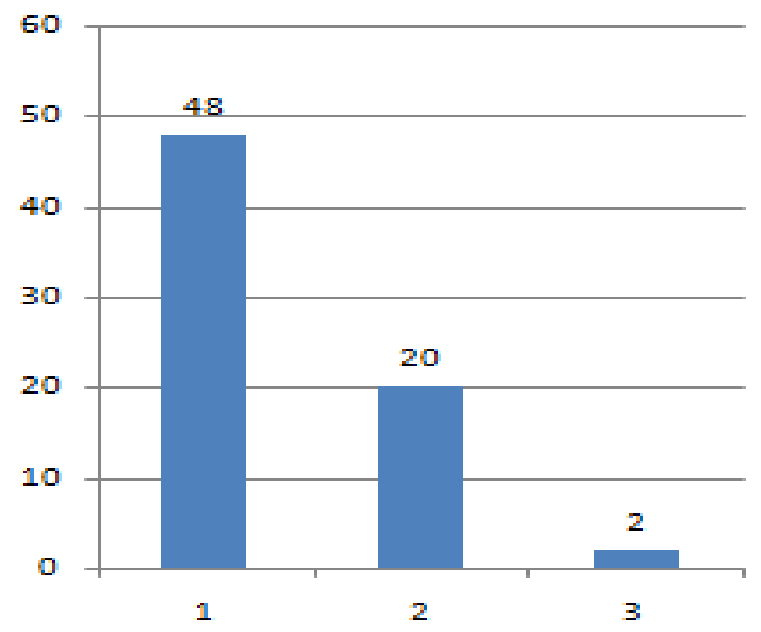

Figure 1: Birth order of the participants

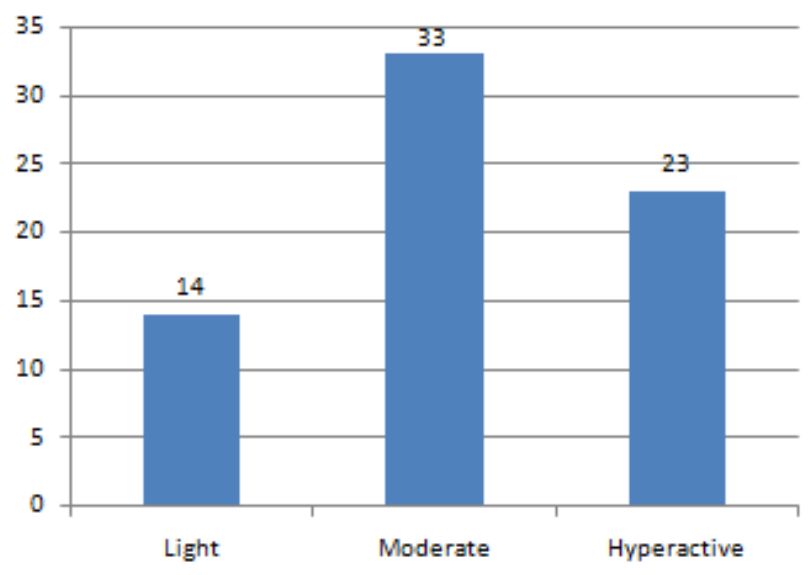

Figure 2: Physical activity of the participants

Problem with Communication skills: As per the study group, majority of autism kids can only obey simple order and they lack in speech \& language. Similarly, they also have repeated stereotype. It was found out that majority of respondents cannot speak using full sentences and they rely only on individual words and cannot apply grammar to construct the sentence. Repeated Stereotype is also common; however, it was also found that many kids have cured their repeated stereotype by right training. Furthermore, majority of kids have fine listening and understanding skills that's why they are able to obey the simple orders and also able to learn new things and try new food. Right communication from parents and care taker can make these kids learn new things gradually over a period of time. Verbal and nonverbal communications are seen to be improved with the growth in age of these respondents.

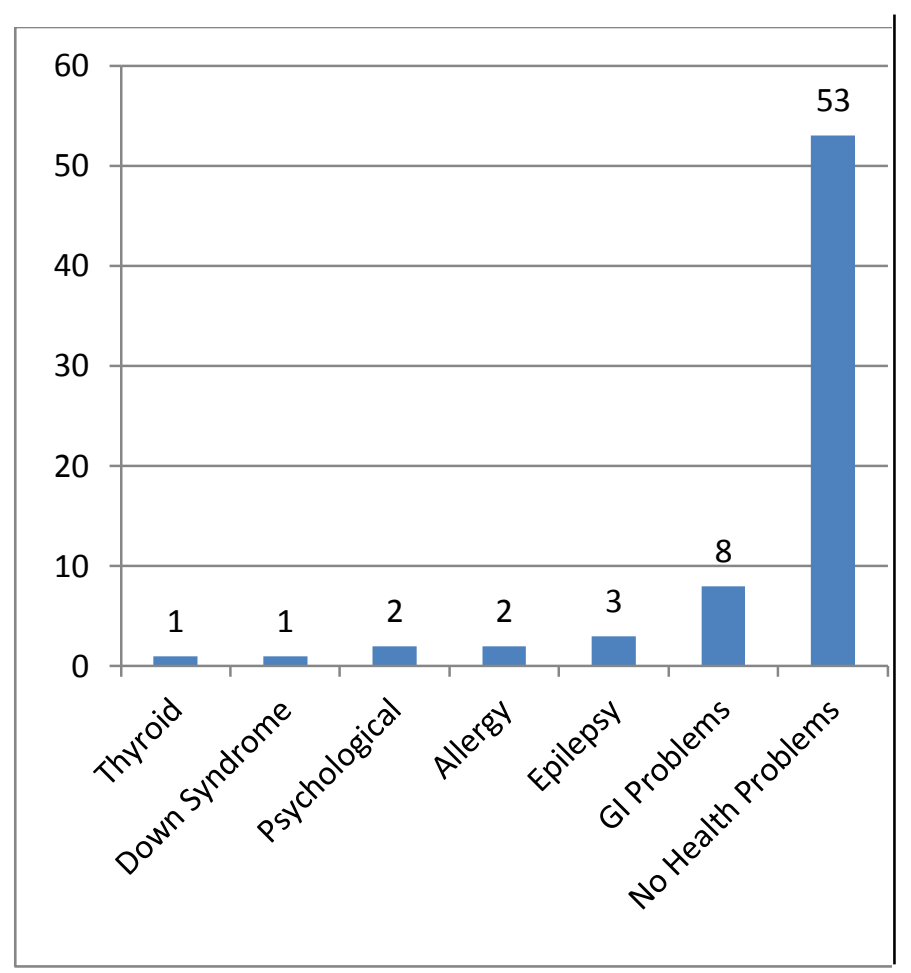

Figure 4: Health problem seen among respondent 


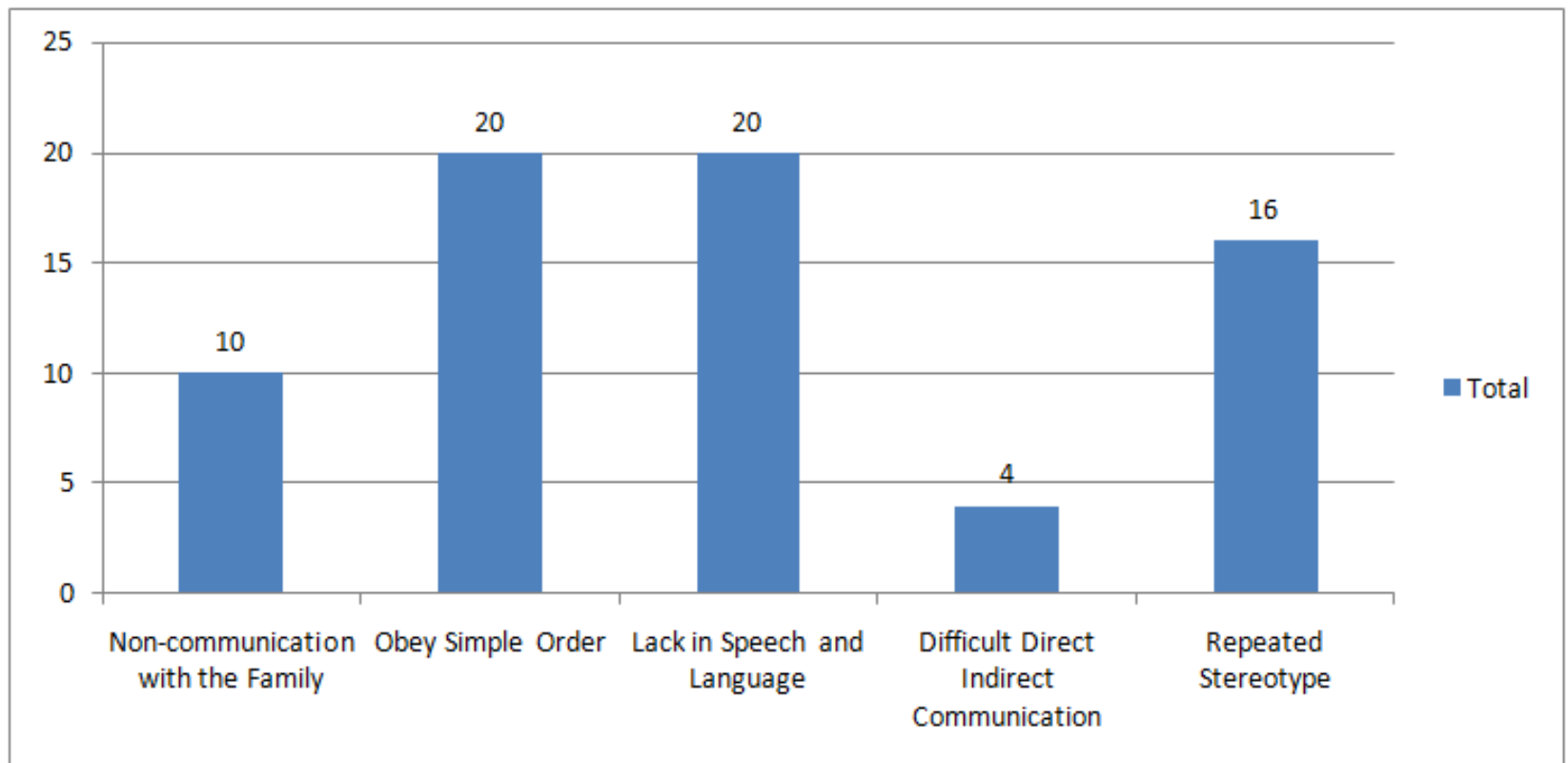

\section{Figure 3: Communication issues of participants}

Health Problems: As per the total sample collected and studied, we could find that majority of autism kids and teenagers do not have any health issues. Over 50\% have reported that no heath problem is seen in them. Very few respondents have shared that their kids were suffering from GI Problems, Epilepsy, Allergy, Psychological, Down Syndrome and Thyroid.

Table 2: BMI status of boys and girls

\begin{tabular}{|l|c|c|c|}
\hline & Boys & Girls & Total \\
\hline $\begin{array}{l}\text { Number of children } \\
\text { assessed: }\end{array}$ & 48 & 22 & $\mathbf{7 0}$ \\
\hline $\begin{array}{l}\text { Underweight (<5th \%ile) } \\
\text { Normal BMI (5th - 85th } \\
\text { \%ile) }\end{array}$ & $4 \%$ & $5 \%$ & $\mathbf{4 \%}$ \\
\hline $\begin{array}{l}\text { Overweight or obese ( } \geq \\
\text { 85th \%ile)* }\end{array}$ & $31 \%$ & $55 \%$ & $\mathbf{3 9 \%}$ \\
\hline \multicolumn{1}{|c|}{ Obese ( $\geq 95$ th \%ile) } & $46 \%$ & $14 \%$ & $\mathbf{3 6 \%}$ \\
\hline
\end{tabular}

BMI status of boys and girls: Among 70 participants, about $4 \%$ individuals were underweight, 39\% normal and 57\% were overweight or obese ( $\geq 85$ th percentile). Out of this $36 \%$ were obese ( $\geq 95$ th percentile) which is shown in table no 2 .

BMI status of autism children with age: Table 3 shows BMI status of children with autism by age group. Here in this study participants of age 3 to 19 years the group created are age group 1-3 years, 4-6 years, 79 years, $10-12$ years, $13-15$ years and 16- 19 years. The highest number of obese children was observed in 4-6 age groups (25) where as the lowest number was seen in age group 1-3 years which comes out to be 2 only.

Overweight and obesity: The figure 4 shows the percentage of overweight and obese children. Fifty-seven percent (57\% i.e 40 ) participants were in overweight/obese group, out of which $36 \%$ i.e, 25 were obese. Which is alarming in number. 
Table 3: BMI status of autism children with age group

\begin{tabular}{|c|r|}
\hline Age group 1 to 3 & $\mathbf{2}$ \\
\hline Obese & 2 \\
\hline Age group4 to 6 & $\mathbf{2 5}$ \\
\hline Normal & 6 \\
\hline Obese & 12 \\
\hline Overweight & 6 \\
\hline Underweight & 1 \\
\hline Age group 7 to 9 & $\mathbf{1 6}$ \\
\hline Normal & 8 \\
\hline Obese & 3 \\
\hline Overweight & 5 \\
\hline Age group 10 to 12 & $\mathbf{1 2}$ \\
\hline Normal & 7 \\
\hline Obese & 3 \\
\hline Overweight & 1 \\
\hline Underweight & 1 \\
\hline Age group 13 to 15 & $\mathbf{8}$ \\
\hline Normal & 4 \\
\hline Obese & 2 \\
\hline Overweight & 1 \\
\hline Underweight & 1 \\
\hline Age group 16 to 19 & $\mathbf{7}$ \\
\hline Normal & 2 \\
\hline Obese & 3 \\
\hline Overweight & 2 \\
\hline Grand Total & \\
\hline
\end{tabular}

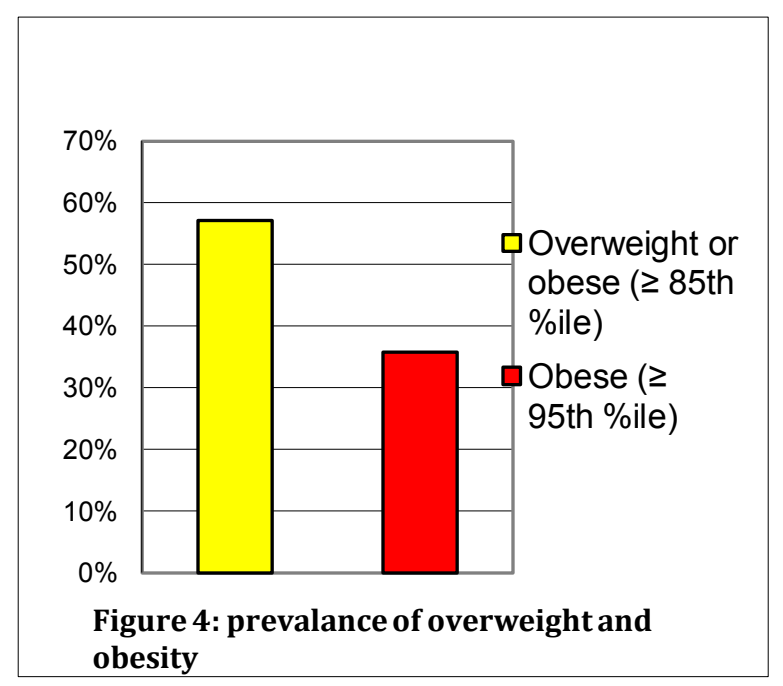

Food selectivity: Determination of food selectivity among autism children is one of the major objectives of this study. Among the 70 total samples collected and studied, 36 individuals (51.43\%) were food selective while 34 individuals (48.57\%) were not food selective. Three domains (refusal, high frequency single food intake and limited repertoire) can be used together or separately to characterize an individual's food selectivity. For the present analysis, the food refusal domain is elected as the measure of food selectivity.

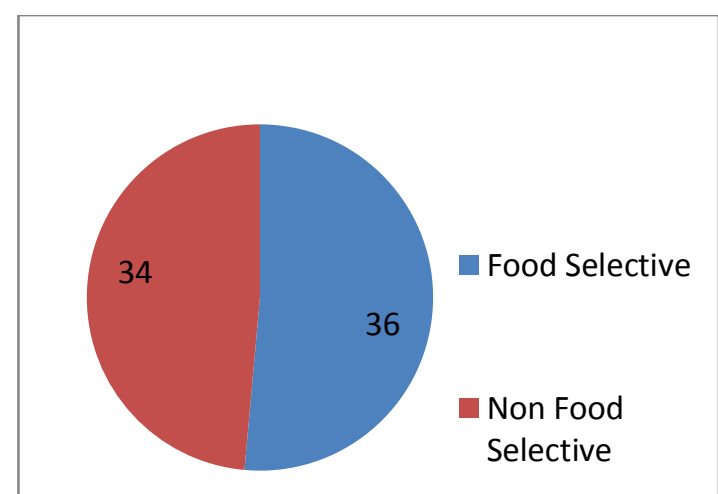

Figure 5: Food Selectivity in participants

Food refusal basis: The basis of food refusal was asked to the parents. The basis of food refusal by their children was not known to $63 \%$ while remaining responded that the child refused food on the basis of temperature and color of the food. It is presented in figure 6.

Meal time behavior: As per the total sample collected and studied, we found that $51.43 \%$ of autism children and adolescent do not have problematic meal time behavior while $48.57 \%$ of them have problematic meal time behavior. It is presented in figure 7 .

Association of various independent variables with the dependent variables: 
Table 4: Association of various independent variables with the dependent variable (BMI)

\begin{tabular}{llll}
\hline Factors & Association with & Chi-square value & P-value \\
& BMI & & \\
\hline Age & Not significant & 6.1147 & 0.106163 \\
\hline Gender & Not significant & 6.070547 & 0.108228 \\
\hline Family income & Not significant & 5.530617284 & 0.13681879 \\
\hline Physical activity & Not significant & 3.284829 & 0.772342 \\
\hline Food Selectivity & Not significant & 4.728664 & 0.192779 \\
\hline Mealtime behavior & Not significant & 1.381022 & 0.709989
\end{tabular}

Table 4 shows the results of association of BMI with the predictive variables. There was no significant association between any of the variables with BMI
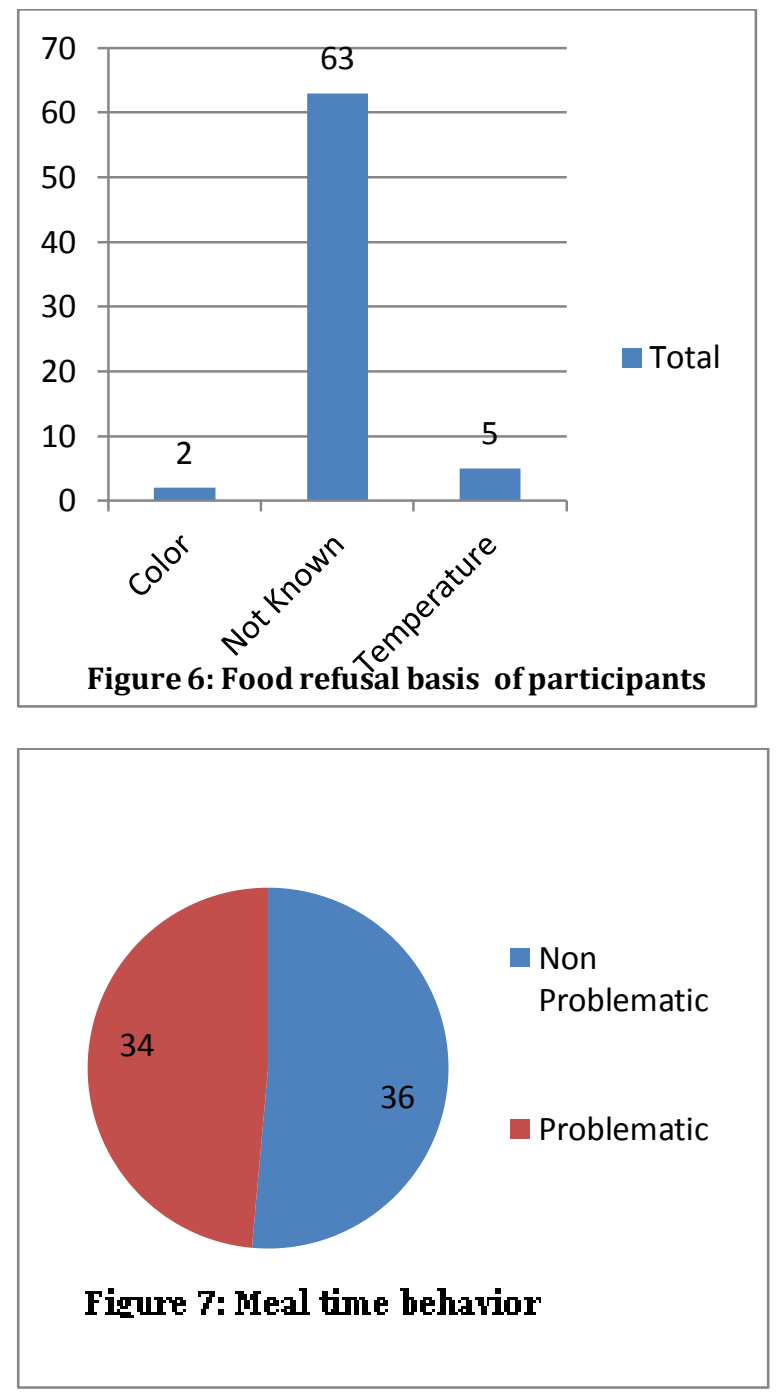

Table 5: Association of Food Selectivity and Mealtime behavior with Obesity

\begin{tabular}{llll}
\hline Factors & $\begin{array}{l}\text { Association- } \\
\text { with } \\
\text { Obesity }\end{array}$ & $\begin{array}{l}\text { Chi } \\
\text { square } \\
\text { value }\end{array}$ & P-value \\
\hline $\begin{array}{l}\text { Food } \\
\text { selectivity }\end{array}$ & Significant & 5.66295 & $0.0173267^{*}$ \\
$\begin{array}{l}\text { Meal time } \\
\text { behavior }\end{array}$ & $\begin{array}{l}\text { Not } \\
\text { significant }\end{array}$ & 1.1438 & 0.284853 \\
\hline
\end{tabular}

\section{*, Significant at $\mathbf{p}<0.05$}

Table 5 shows the results of association between food selectivity and mealtime behavior with overweight/obesity. Mealtime behavior showed no significant association with obesity while food selectivity showed significant association with obesity at $\mathrm{p}<0.05$.

\section{Comparisons of Estimated Calorie Intake of} Respondents with Recommended Daily Allowances by Indian Council of Medical Research (ICMR):

Table 6 shows the comparisons of estimated calorie intake of respondents with ICMR recommended daily allowances. In this study the one sample t- test was used for the total calorie intake of children by their age group and analyzed whether they meet the requirement set by ICMR or not. 
Table 6: Comparisons of Estimated Calorie Intake of Respondents with Recommended Daily Allowances (RDA)

$\begin{array}{lllll}\text { Variable } & \text { Frequency (n) } & \text { Estimated Total Calorie } & \text { ICMR RDA } & \text { P-Value }\end{array}$

$$
\text { Mean } \pm \text { SD }
$$

\begin{tabular}{llrrrr}
\hline Age Group & Sex & & & \\
$\mathbf{1 - 3}$ & Both & 2 & $1739 \pm 239$ & 1060 & 0.04397 \\
$\mathbf{4 - 6}$ & Both & 25 & $1936.72 \pm 230.21$ & 1350 & $<0.001$ \\
$\mathbf{7 - 9}$ & Both & 16 & $2072.06 \pm 183.74$ & 1690 & $<0.001$ \\
$\mathbf{1 0 - 1 2}$ & Male & 10 & $2223.2 \pm 413.77$ & 2190 & $<0.001$ \\
& Female & 2 & $1937.5 \pm 65.5$ & 2010 & 0.01076 \\
$\mathbf{1 3 - 1 5}$ & Male & 4 & $2241.5 \pm 386.49$ & 2750 & 0.00105 \\
& Female & 4 & $2298.75 \pm 205.44$ & 2330 & 0.000150 \\
$\mathbf{1 6 - 1 9}$ & Male & 5 & $2913.8 \pm 106.29$ & 3020 & $<0.001$ \\
\hline & Female & 2 & $2099.5 \pm 121.53$ & 2440 & 0.0184 \\
\hline
\end{tabular}

Calorie intake of respondents was compared with ICMR RDA. There were 2 participants in age group 1-3 years and mean calorie intake was found to be 1739 with SD of 239. Similarly, the mean calorie intake of age group 4-6 was found to be 1936 (SD 230.21) and that of 7-9 was found to be 2072.06 (SD 183.74). Among 10 numbers of boys aged 10 12 years, mean calorie intake of 2223.2 with

Table 7: Male and Female RDA Comparison

\begin{tabular}{llcc}
\hline \multicolumn{1}{c}{ Sex } & $\begin{array}{c}\text { Age in } \\
\text { years }\end{array}$ & Below RDA & $\begin{array}{c}\text { RDA Equal } \\
\text { or above }\end{array}$ \\
\hline Male & $1-3$ & 0 & 1 \\
\hline Female & $1-3$ & 0 & 1 \\
\hline Male & $4-6$ & 0 & 18 \\
\hline Female & $4-6$ & 2 & 5 \\
\hline Male & $7-9$ & 2 & 9 \\
\hline Female & $7-9$ & 3 & 2 \\
Male & $10-12$ & 5 & 5 \\
Female & $10-12$ & 1 & 1 \\
Male & $13-15$ & 3 & 1 \\
\hline Female & $13-15$ & 1 & 3 \\
Male & $16-19$ & 0 & 5 \\
Female & $16-19$ & 1 & 1 \\
Total & & 18 & 52 \\
\hline
\end{tabular}

SD of 413.77 and was observed to be 1937.5 KCal (SD 65.5) for girls.

The mean calorie intake of male and female of age group 13-15 was found to be 2241.5 (SD 386.49) and 2298.75 (SD 205.44) respectively. the mean calorie intake of male and female participants of age group 16-19 years was observed to be 2913.8 (SD 106.29) and 2099.5 (SD 121.53) There was significant difference in mean calorie intake by all the age groups than the calorie set intake by ICMR as p-value was $<0.05$ in all age group.

Table 7 shows that out of 70, 20 participants consumed below RDA while 50 participants consumed equal or above RDA requirements.

\section{Macronutrient intake of Children with ASD:}

Table 8 demonstrates that almost all of the macronutrient intakes were within the acceptable macronutrient distribution range for all age groups, besides fat. Age group 1-3 years consumed higher carbohydrate intake with mean percentage of $67.12 \%$. 
Table 8: Mean Macronutrient intake of Children with ASD

\begin{tabular}{lllllll}
\hline $\begin{array}{l}\text { Mean } \\
\downarrow\end{array}$ & $\mathbf{1 - 3}$ years & 4-6years & 7-9years & $\mathbf{1 0 - 1 3}$ years & $\mathbf{1 3 - 1 5}$ years & $\mathbf{1 6 - 1 9}$ years \\
\hline Protein,g & & & & & & \\
Protein \% KCal & 200.05 & 119.14 & 361.83 & 149.28 & 171.69 & 189.51 \\
CHO,g & $22.68 \%$ & 25.02 & $25.2 \%$ & $27.36 \%$ & $30.53 \%$ & $27.78 \%$ \\
CHO \% KCal & 290 & 315.32 & 305.50 & 321.42 & 327.50 & 405.86 \\
Fat, g & $67.12 \%$ & 64.66 & $58.85 \%$ & $59.14 \%$ & $57.52 \%$ & $61.22 \%$ \\
Fat, KCal & 19.66 & 10.31 & 68.02 & 32.53 & 30.38 & 33.30 \\
\hline
\end{tabular}

\section{DISCUSSION}

The present study included 70 participants ( 49 males and 21 females) under the respondents with mean age of 8.95 years. The children aged 3 years were 2, 4-6 years were 25, 7-9 age group were 16, 10-12 aged children were 12 in number. Among 15 adolescents, 8 were between 13-15 years and 7 were between 16-19 years. Study subjects were from all categories, majority being Brahmin (50\%) followed by Newar (17.14\%). Among the participants, majority of them were Hindu (97.14\%). The majority of the participants had family size of 4 (35.71\%) followed by 3 and 5 (24.29\% each).The birth order of majority of the children $(68.57 \%)$ was found to be 1 i.e, majority of them were the first child of the parents. 20 of them $(28.57 \%)$ were the 2 nd child while only 2 of them were the third child of the parents.

In the present study most of the parents of the children with autism were a Bachelor's graduate. about $35.71 \%$ of the father's education level was a Master's. Only 2.86\% and $1.43 \%$ of father and mother were illiterate. Whereas according to NDHS report $2011,41 \%$ of women had never been school, $23 \%$ ad an incomplete primary education, $6 \%$ had completed primary school but not continued on to the next level of schooling, and $25 \%$ have some secondary school and education [13]. This is a very good sign because well-educated parents can better take care of their children's food habits. In this study it was well observed that the majority of the literate parents were open to new information regarding the food habit of their children.

\section{Autism related characteristics}

Figure 2 shows the physical activity status of the children with autism. The current study observed that most (47.14\%) of the participants had moderate physical activity level followed by extremely active (32.86\%). In $32.86 \%$ of the extremely active behavior could be due to attention deficit hyperactive disorder (ADHD). Recent studies indicate that co-occurrence of clinically significant ADHD and autistic symptoms is common, and that some genes may influence both disorders [14]. Figure 3 shows the information regarding the communication behavior. Most of the participants obey simple order (28.57\%). According to the parents this improvement in behavior might be probably due to the therapies that have been being given to the children. Also $28.57 \%$ of them lack in speech and language. The common health problems that are found to be associated with children in the current study were gastrointestinal problems (75.71\%) and epilepsy (4.29\%). However as per the parents, majority of them $(75.71 \%)$ had no health issues. About $90 \%$ of the participants were not taking any medicines currently. While $10 \%$ were taking some medicines especially for epilepsy, for ADHD and antipsychotics. About $24.29 \%$ of the participants were being given food supplements. The food 
supplements majorly consumed included fish oil and vitamin syrups.

\section{BMI}

Among 70 participants, about 4\% individuals were underweight, 39\% normal and 57\% were overweight or obese ( $\geq 85$ th percentile). Out of this $36 \%$ were obese ( $\geq 95$ th percentile). This finding is consistent with other findings in CWA. In a retrospective chart review study done by Curtin et al of children aged 3-18 years, the overall prevalence of at-risk-for-overweight was $35.7 \%$ and prevalence of overweight was $19 \%$ [4]. A survey was conducted in Iran in 113 children and adolescents (boys $=90$, girls = 23) aged 7-14 years old attending autismspecific schools in Tehran. The findings revealed that $50.4 \%$ of these young children were in the limits of normal weight, but $8.7 \%$ were underweight, $13.3 \%$ were overweight, $11.5 \%$ were obese, and $15.9 \%$ were severely obese. The study concluded that with increase in the prevalence of ASD, there was found a growing rate of obesity in these children [15].

Similarly in a study conducted by Egan et al, Rates of overweight and obesity in children with ASD were found to be above nationally representative prevalence estimates for children. Among children with autistic disorder, $17.16 \%$ had a body mass index (BMI) percentile in the overweight range and $21.89 \%$ had a BMI percentile in the obese range [16]. Another study also revealed similar findings in children age 2 to 17 years, $33.6 \%$ were overweight and $18 \%$ were obese. Compared with a general US population sample, rates of unhealthy weight were significantly higher among children with ASDs ages 2 to 5 years [17].
A review was done by Balogun Femi, 2016, to find out the evidence on obesity and overweight prevalence in the childhood ASD population from relevant studies using a computer generated database. Compared to an obesity rate of $17 \%$ in typically developing children from the CDC's National Health and Nutrition Examination survey (2011-2014); eight of the eleven studies included in this review reported higher obesity rates among children with ASD. The highest rate was 30\% and the lowest was 10\% [18].

In a study conducted by Grondhius Sabrina,2014, the sample comprised children from the United States and Canada who visited a hospital or clinic that was part of the Autism Treatment Network. In this sample, $32.9 \%$ of the children were overweight and $17.3 \%$ were obese [19]. Similarly in a study conducted by Nor et al. in Malasiya, 2019, on 151 ASD children aged 2-18 years, the prevalence of overweight (BMI $\geq 85$ th to $<95$ th percentiles) was $11.3 \%$ and the prevalence of obesity (BMI $\geq 95$ th percentile) was $21.9 \%$. The prevalence of obesity and overweight is high among Malaysian ASD children and adolescents which is similar to our findings. Older child age, high maternal BMI, older paternal age, low physical activity, low likelihood of food refusal and high likelihood of food selectivity were found to be risk factors for high BMI in these children [20].

In a study conducted in Egypt by Megud et al, all the 80 participated children were overweight: the BMI - $\mathrm{z}$ scores of group 1 were $0.95 \pm 0.04$ and $0.4 \pm 0.3$ and for group 2 were $0.9 \pm 0.05$ and $1.6 \pm 0.5$ for both sexes (boys and girls) respectively and showed high significant in Group 2. The cutoff point for the BMI- z score was \pm 0.2 [21]. 
Dietary studies consistently reveal that hyperactive children eat more sugar than other children [22]. The problem is not sugar itself but the forms it comes in, the absence of a well-balanced diet overall. In any case, when a child is regularly snacking on refined carbohydrates, sweets, chocolate, fizzy drinks, juices and little or no fiber to slow the glucose absorption, the levels of glucose in their blood will fluctuate continually and trigger wild fluctuations in their levels of activity, concentration, focus and behavior. This signifies that the high consumption of refined carbohydrates not only affects the weight status of CWA but also has effect on their behavior.

Childhood obesity has been associated with adverse health outcomes, including insulin resistance, diabetes, heart disease, and certain cancers [23]. It is very difficult for childhood obesity management especially in case of ASD. The first is that physical limitations associated with ASD like subtle difficulties with muscle tone and coordination problems; hinder participation and engagement in existing curriculum and community based exercise programs as designed for typically developing children. The core social interaction and communication deficits of ASD limit access to and participation in physical activity programs in and out of school. So it is very necessary to control the weight in CWA as they were not aware of the amount of food they eat. Most of the parents mentioned that their child does binge eating so the parents themselves keep the foods away from the child to prevent from being overweight and obese. Parents' attention and care play an important role in maintaining normal weight in CWA.

\section{Food Selectivity}

Among 70 participants, 36 individuals (51.43\%) were food selective while remaining $34 \quad(48.57 \%)$ were not food selective. Our finding is relatable to most of the study findings that have been done to determine food selectivity in autism. MauriBauset [24] and colleagues conducted a systematic review of the scientific literature from 1970 to 2013 related to food selectivity in ASD. More recent studies have confirmed this pattern. Williams et al [25] indicated that overall $67 \%$ of parents complained of food selectivity, it being determined by the following factors: texture (69\%), appearance (58\%), taste (45\%), smell (36\%), and temperature $(22 \%)$, as well as reluctance to try new foods (69\%) and a small repertoire of accepted foods (60\%). However in our study, the basis of food refusal was not known to many parents (63\%). Few parents observed that their children refused foods given to them because of temperature (5\%) and color of food (2\%).

Similarly, Schreck et al found that $72 \%$ of parents reported their children having a limited repertoire of foods and 57\% reported food refusal. Foods were mainly rejected because of their presentation, the use of certain utensils, or different types of food being served on the same plate (48.6\%) [26]. There are other study which concluded that there is an scientific consensus supporting an association between food selectivity and autism spectrum disorders [24].

Similarly, in a study conducted by Hubbard and colleagues in 2014, children with ASD were significantly more likely to refuse foods based on texture/consistency $(77.4 \%$ versus $36.2 \%)$, taste/smell (49.1\% versus $5.2 \%)$, mixtures $(45.3 \%$ versus $25.9 \%)$, brand $(15.1 \%$ versus $1.7 \%)$, and shape $(11.3 \%$ 
versus $1.7 \%$ ) than the typically developing (TD) children. No differences between groups were found for food refusal based on temperature, foods touching other foods, or color [27]. William et al. conducted a study to examine food selectivity in children with ASD by asking parents about their child's eating habits. The researchers gathered information from 100 parents of children having ASD from 22 months to 10 years. Parents believed that food selectivity was influenced by: texture (69\%), appearance (58\%), taste (45\%), smell (36\%), and temperature (22\%) [25]. The most frequent eating and oral behavior problems to be observed were reluctance to try new foods (69\%), resistance to medication (62\%), eating less foods (60\%), mouthing objects (56\%) and traditional eating (46\%). Based on the findings of this study, sensitivity towards foods appearance to be an underlying reason why children with ASDs were classified as picky eaters [28].

Few studies have determined the prevalence of food selectivity in children with an ASD compared to NIC (Neuro impaired comparison) and TD children. Nicole Mc Donald conducted a study in 2014. The results illustrated that the ASD group were significantly more food selective $(46 \%)$ when compared to NIC (31\%) and TD (26\%)[7].

Bandini and colleagues have done numerous study related to food habits of children with Autism anfood selectivity in autism and provided significant information regarding food selectivity pattern in ASD. In one of the studies conducted by Bandini et al, the findings suggest that food selectivity is more common in children with ASD than in typically developing children. Food selectivity was compared between 53 children with ASDs and 58 typically developing children ages 3-11 years. Children with ASDs exhibited more food refusal than typically developing children $(41.7 \%$ vs. $18.9 \%$ of foods offered, $\mathrm{p}<0.0001$ ) [8].

Bandini et al conducted another study to find out if food selectivity in young age still persists or not in adolescence. The study was conducted in 18 children at two time points (mean age $=6.8$ and 13.2) with autism and examined the changes in food selectivity. The children's food refusal improved between baseline and follow-up. The overall percentage of foods refused of those offered decreased from $47 \%$ at baseline to $31 \%$ at follow-up $(p=0.005)$. Fruit and vegetable refusal showed similar decreases between baseline and follow-up. The absolute number of foods refused also declined; participants refused an average of 51 foods at baseline and 33 foods at follow-up $(p=0.01)$. The number of foods 'not offered' did not change significantly between baseline and follow-up (22 vs. 27, p=0.43). At baseline, 15 participants $(83 \%)$ refused $>33 \%$ of foods offered and met our definition of high food selectivity. At follow-up, 7 (39\%) of these participants no longer had high food selectivity. Eight participants (44\%) had high food selectivity at baseline and at follow-up. Three children were below the threshold for high food selectivity at both time-points [29].

\section{Meal time behavior}

The current study determined that among 70 participants, $51.43 \%$ of autism children and teenagers do not have problematic meal time behavior while $48.57 \%$ of them have problematic meal time behavior. Many studies have indicated that people with autism have mealtime problems. In a study conducted in Canada by Nadon et al [30], in 
CWA and their siblings (3-12 years of age), results demonstrate that problems related to eating and mealtimes were significantly more common in children with ASD than their typically developing siblings even when adjusted for age and sex. Nevertheless, both groups experience eating problems and none of the problems encountered were exclusive to children with ASD, aside from some oralmotor difficulties. More children with ASD showed behaviors such as drooling, coughing, gagging, and vomiting during mealtimes than their siblings and some parents interpreted these motor signs as the cause of their children's feeding problems [30]. Similarly, in the study conducted by Palta and Saxena in India, 2014, aggressive behavior, specific food selection or refusal and moving around during mealtime were some of the behaviors observed among the Autistic children i.e, CWA had problematic meal time behavior [11].

A study conducted was by Curtin and colleagues, 2016, among 53 children with ASD and 58 typically developing (TD) children ages 3-11 years. Compared to TD children, children with ASD were more likely to have high food selectivity, and their parents reported more mealtime behavior problems. High food selectivity was associated with mealtime behavior problems in both groups. Interventions to reduce food selectivity may lead to decreases in mealtime behavior problems [31]. Similarly another study was conducted in Hong Kong to determine mealtime behavioral problems. As expected, Hong Kong Chinese preschoolers with Autism had poor food variety scores. Some of them appeared to be flexible in their food choices, while others were much more limited and seemed to restrict their intake. There was a high prevalence of limited variety of food behavior such as unwillingness to try new food, a dislike of certain food and refusing to eat them [10].

In the present study, out of 70 individuals, 36 had no problematic meal time behaviors. This might be again due to the OT that have been being given to the children in Autism Care Nepal Society. Many of the parents, when asked about the meal time behaviors about their children, said that before there were some problems during meal time but has improved substantially after the therapies and training to the children. However, remaining 34 had the problematic meal time behavior. Children with Autism demonstrate a wide range of more problematic meal time behaviors than the general population [31]. The reason for problematic eating habits among children with Autism has not been thoroughly investigated, but rigid adherence to rituals and routines seen as a core feature of autism is one of the hypothesized explanation. Sensory dysfunction may also play a role in problematic meal time behaviors [32]. Children with ASD have problems correctly identifying taste and olfactory sensations suggesting that over or under responsiveness to sensory stimuli may also contribute to the high prevalence of feeding difficulties and meal time problems among this population [33]. This is an area that requires further investigation in larger population of this developmental disorder.

\section{Association between independent variables with the dependent variable (BMI)}

Table _ shows no significant association between BMI and all the predictive variables as $p$ value is $>0.05$. No significant association was found between obesity and mealtime behavior. However significant association was found between obesity and food selectivity. Previous literature and the 
current dataset demonstrated that children with an ASD, who were food selective, preferred energy dense foods which may contribute to the development of overweight and/or obesity over a period of time [7, 31]. Greater detail and more research were needed to better understand the correlation between BMI status and food selectivity in children with an ASD.

\section{Dietary Intake}

There was significant difference in mean calorie intake by all the age groups than the calorie set intake by ICMR as p-value was $<0.05$ in all age group. For most of the age groups, the mean calorie intake was higher than the RDA. From 24 hour dietary recall method, most of the children were found to have consume more energy dense food especially high sugar containing foods like chocolates, ice-cream, sweets, cold drinks, canned juices. The present study specifically observed that younger children were consuming high calorie containing foods (from 3-6 years ) which might lead to overweight/obesity in adolescence if they continue to eat in this pattern while many of them were already overweight/obese. The mean macronutrient consumption for all age groups was within the acceptable range for carbohydrate (45-65\%) and protein (10$30 \%$ ) besides the fat. Intake of fat supplies essential fatty acids (EFA) and aids in the absorption of fat-soluble vitamins A, D, E and $\mathrm{K}$. It is also a substrate for the production of hormones and mediators. Fat, especially in infancy and early childhood, is essential for neurological development and brain function $[34,35]$. The low intake of fat may make the children vulnerable to risk of unsatisfactory intakes of fat-soluble vitamins.

In a study conducted in Egypt by Megud et all in 80 children [21], participants were divided into 2 groups group 1 (aged 3-5) and group 2 (aged 6-9 years). The results revealed that children with autism consumed average amount of calories: group $1=83 \%$ and group $2=94 \%$ of RDAs for their age. Carbohydrates also were within average intake (54-60\%) of the total caloric intake but shifted towards the upper-end of normal range for the older age group (6-9 years old). Protein intakes were slightly high when compared with the expected RDA but within normal range when calculated as a percentage (\%) of total energy intake. But fat intake was more than the RDAs for their age with high saturated fats content which was different from the current study [21].

\section{CONCLUSION}

From The present study is the first in Nepal to examine food selectivity and mealtime behavior problems in children with autism. This results present that majority of CWA were overweight/obese. The results should provide a good insight that most of the children were in the state of over-intake of total calorie. The findings of the present study provide a new insight into the food habit of autism children in Nepal. The study describes the food selectivity, meal time behavior problem, nutrient intake and weight status in children and teen with Autism. The data from this study suggest that just as overweight/obesity is a problem for general population, overweight/obesity also represents a problem among children with ASD. Furthermore, the burden of managing potential co-morbidities associated with obesity may be particularly problematic for children with developmental disorders and their families, and may limit options for living independently in adulthood. Therefore, this area of research warrants focused attention and effort. There were no reports of food 
selectivity, meal time behavior problems in this particular population in Nepal. While preliminary and purely descriptive, these findings provide a clearer understanding of feeding problems in this population. Given the accumulating evidence of food selectivity and meal time problems in children with ASD, it is critical that future research explore solutions for improved food choices among these population with ASD to improve health outcomes and healthy lifestyle choices.

Future research should develop and examine the effectiveness of interventions tailored to the needs of families of children with ASD, including the effectiveness of interventions for food selectivity and modifying mealtime behaviors in this population. Considering the additional implications of the high rates of overweight/obesity, many of these children were in need of intervention and screening for nutritional deficiencies should be extended to children who were overweight as well as underweight.

Recommendation for practices: These findings should provide insight to professionals and dietitians when they work with children with an ASD who were food selective and have meal time behavior problems. Professionals should be aware that food selectivity is more common in children with an ASD. The data clearly illustrate that children with an ASD were more likely to consume energy dense foods which can have implications on weight and nutritional status long-term. The findings of this study might present a need to establish the treatment programs designed to help children with ASD to improve their feeding behaviors and development of research towards creating a specific treatment pans would be valuable. Parents and other family members play a major role in understanding, assessing, and developing an effective intervention plan for selective eating in a child with ASD. Family routines and habits influence the eating behaviors of children and positive interactions among family members may result in increased participation in mealtime, including appropriate social behaviors and eating a variety of foods. Assessments and interventions for children with ASD and selective eating should include a nutritional component, with a registered dietitian as part of the interdisciplinary team to ensure adequate nutrition. A pediatric gastroenterologist can offer valuable insight on how GI issues may play a role in the diagnosis and treatment of selective eating. Primary care physicians can also play an important role in monitoring progress and health over time.

Recommendation for future research: While the entire ASD population's health is worthy of extensive research, it is critical to conduct feeding-related studies focusing on young children with ASD, as this is the age when rapid and dramatic growth is occurring and nutritional deficits may be more detrimental. This type of research would be not only beneficial to individuals with ASD, caregivers, clinicians, and dietitians but also educational to society as a whole.

\section{ACKNOWLEDGEMENTS}

Authors would like to thank all the staff of Autism Care Nepal Society for their support in conducting this study.

\section{REFERENCES}

1. Faras, H., Al Ateeqi, N., \& Tidmarsh, L. (2010). Autism spectrum disorders. Annals of Saudi medicine 2010; 30(4): 295-300.

2. Speaks, A. What is autism. Retrieved from https://www.autismspeaks.org/what-autism on November, 17, 2018.

3. Balikci, OS, \& Ciyiltepe, M. (2017). Feeding problems of children with Autism People. 
International Journal of Social Sciences 2017; 3(1):Special issue Retrieved from https://grdspublishing.org/index.php/people /article/view/477

4. Curtin, C., Bandini, L. G., Perrin, E. C., Tybor, D. J., \& Must, A. Prevalence of overweight in children and adolescents with attention deficit hyperactivity disorder and autism spectrum disorders: a chart review. BMC pediatrics 2005; 5(1): 48.4 DOI: https://doi.org/10.1186/1471-2431-5-48

5. Geraghty, M. E., Depasquale, G. M., \& Lane, A. E. Nutritional intake and therapies in autism: A spectrum of what we know: Part 1. ICAN: Infant, Child, \& Adolescent Nutrition 2010; 2 (1): 62-69.

6. Jena Leigh Altenburger, B. The quality of nutritional intakes in children with autism: A prospective study. The Ohio State University., 2010.

7. Withrow-McDonald, N. A. . Food selectivity and weight status in children with an Autism Spectrum Disorder (ASD). Colorado State University, 2014.

8. Bandini, L. G., Anderson, S. E., Curtin, C., Cermak, S., Evans, E. W., Scampini, R., et al.. Food selectivity in children with autism spectrum disorders and typically developing children. The Journal of pediatrics, 2010;157(2):259-264.

9. Cherif, L., Boudabous, J., Khemekhem, K., Mkawer, S., Ayadi, H., \& Moalla, Y. Feeding problems in children with autism spectrum disorders. Journal of Family Medicine 2018; 1(1):30-39.

10. Chan, D., YU, C. W. C., So, H., Chan, S., \& Tsang, $\mathrm{N}$. Mealtime behavioral problems in Hong Kong Chinese preschoolers with autism spectrum disorder. Journal of Psychological Abnormalities 2016; S1. Retrieved from http://dx.doi.org/10.4172/jpab.S1-004.

11. Palta, A., \& Saxena, R. Meal Time Behavior of Children with Autism. Aggressive behavior 2015; 37(9.8): 52-57.

12. DeMand, A., Johnson, C., \& Foldes, E. Psychometric properties of the brief autism mealtime behaviors inventory. Journal of autism and developmental disorders 2015; 45(9):2667-2673

13. MOHP,2011 Ministry of Health, Nepal; New ERA; and ICF. Nepal Demographic and Health Survey 2016. Kathmandu, Nepal: Ministry of Health, Nepal, 2017.

14. Reiersen, A., \& Todd, R. (2008). Co-occurrence of ADHD and autism spectrum disorders: phenomenology and treatment. Expert review of neurotherapeutics 2008; 8(4), 657-669.
15. Memari, A. H., Kordi, R., Ziaee, V., Mirfazeli, F. S., \& Setoodeh, M. S. Weight status in Iranian children with autism spectrum disorders: Investigation of underweight, overweight and obesity. Research in Autism Spectrum Disorders 2012; 6(1): 234-239.

16. Egan, A. M., Dreyer, M. L., Odar, C. C., Beckwith, M., \& Garrison, C. B. (2013). Obesity in young children with autism spectrum disorders: prevalence and associated factors. Childhood Obesity, 9(2), 125-131.

17. Centre for disease Control\& Prevention (CDC). BMI-Body Mass Index: About BMI for children and teens, Retrieved on May, 7, 2018 from

https://www.cdc.gov/healthyweight/assessin $\mathrm{g} / \mathrm{bmi} /$ childrens bmi/about childrens bmi.ht ml.

18. Balogun, F. Prevalence and correlates of obesity in childhood autism spectrum disorders: A literature review. Journal of Psychiatry 2016; 19: 385. doi:10.4172/23785756.1000385

19. Grondhuis, S. N.. Overweight and Obesity in Children with Autism Spectrum Disorders: Findings Consistent with Typically Developing Children. The Ohio State University, 2014.

20. Kamal Nor, N., Ghozali, A. H., \& Ismail, J. Prevalence of overweight and obesity among children and adolescents with autism spectrum disorder and associated risk factors. Frontiers in pediatrics 2019; 7: 38. doi: 10.3389/fped.2019.00038

21. Meguid, N., Anwar, M., Zaki, S., Kandeel, W., Ahmed, N., \& Tewfik, I. Dietary patterns of children with autism spectrum disorder: a study based in Egypt. Open access Macedonian journal of medical sciences 2015; 3(2): 262267.

22. Johnson, R. J., Gold, M. S., Johnson, D. R., Ishimoto, T., Lanaspa, M. A., Zahniser, N. R., et al.. Attention-deficit/hyperactivity disorder: is it time to reappraise the role of sugar consumption? Postgraduate medicine 2011; 123(5): 39-49.

23. Dhaliwal, K. K., Orsso, C. E., Richard, C., Haqq, A. M., \& Zwaigenbaum, L. Risk factors for unhealthy weight gain and obesity among children with autism spectrum disorder. International Journal of Molecular Sciences 2019; 20(13): $3285 . \quad$ DOI: 10.3390/ijms20133285

24. Marí-Bauset, S., Zazpe, I., Mari-Sanchis, A., Llopis-González, A., \& Morales-Suárez-Varela, M. Food selectivity in autism spectrum disorders: a systematic review. Journal of child neurology 2014; 29(11):1554-1561.

25. Williams KE, Gibbons BG, Schreck KA. Comparing selective eaters with and without 
developmental disabilities. J Dev Phys Disabil. 2005; 17:299-309.

26. Schreck, K. A., \& Williams, K. (2006). Food preferences and factors influencing food selectivity for children with autism spectrum disorders. Research in developmental disabilities 2006; 27(4): 353-363.

27. Hubbard, K. L., Anderson, S. E., Curtin, C., Must, A., \& Bandini, L. G. A comparison of food refusal related to characteristics of food in children with autism spectrum disorder and typically developing children. Journal of the Academy of Nutrition and Dietetics 2014; 114(12): 1981-1987.

28. Theresa, L., Timmons, M., Hogan, K., Jacobson, H., Lebarron, R., \& Narzabal, S. Picky Eating and the Associated Nutritional Consequences. J. Food Nutr. Disord 2017; 6:3. doi: 10.4172/2324-9323.1000227

29. Bandini, L., Curtin, C., Phillips, S., Anderson, S. E., Maslin, M., \& Must, A. Changes in food selectivity in children with autism spectrum disorder. J Autism Dev Disord. 2017; 47(2): 439-446. doi: 10.1007/s10803-016-2963-6

30. Nadon, G., Feldman, D. E., Dunn, W., \& Gisel, E.. Mealtime problems in children with autism spectrum disorder and their typically developing siblings: A comparison study. Autism 2011; 15(1):98-113.

31. Curtin, C., Jojic, M., \& Bandini, L. G. Obesity in children with autism spectrum disorders. Harvard review of psychiatry 2014; 22(2): 93103.

32. Stough, C. O., Gillette, M. L. D., Roberts, M. C., Jorgensen, T. D., \& Patton, S. R.. Mealtime behaviors associated with consumption of unfamiliar foods by young children with autism spectrum disorder. Appetite 2015; 95:324-333.

33. Roth, S. Feeding Problems in Individuals with Autism and Developmental Disabilities. Barnard College, 2012.

34. Milner, J. A., \& Allison, R. G. The role of dietary fat in child nutrition and development: summary of an ASNS workshop. The Journal of nutrition 1999; 129(11):2094-2105.

35. Vobecky, J., Vobecky, J., \& Normand, L.. Risk and benefit of low fat intake in childhood. Annals of nutrition and metabolis 1995; 39(2):124-133.

\section{Correspondence to:}

Prof. Kshitiz Upadhyay Dhungel

Department of Nutrition and Dietetics

College of Applied Food and Dairy Technology (CAFODAT)

Baneshwor, Kathmandu, Nepal

kistiz2003@gmail.com 